\title{
A PRÜFER TRANSFORMATION FOR THE EQUATION OF THE VIBRATING BEAM
}

BY

\author{
D. O. BANKS AND G. J. KUROWSKI
}

ABSTRACT. In this paper, the oscillatory properties of the eigenfunctions of an elastically constrained beam are studied. The method is as follows.

The eigenfunction and its first three derivatives are considered as a fourdimensional vector, $\left(u, u^{\prime}, p u^{\prime \prime},\left(p u^{\prime \prime}\right)^{\prime}\right)$. This vector is projected onto two independent planes and polar coordinates are introduced in each of these two planes. The resulting transformation is then used to study the oscillatory properties of the eigenfunctions and their derivatives in a manner analogous to the use of the Prüfer transformation in the study of second order Sturm-Liouville systems. This analysis yields, for a given set of boundary conditions, the number of zeros of each of the derivatives, $u^{\prime}, p u^{\prime \prime},\left(p u^{\prime \prime}\right)^{\prime}$ and the relation of these zeros to the $n-1$ zeros of the $n$th eigenfunction. The method also can be used to establish comparison theorems of a given type.

1. The main purpose of this paper is to study the oscillatory properties of the solutions and their derivatives of the eigenvalue problem

$$
\begin{gathered}
\left(p(x) u^{\prime \prime}\right)^{\prime \prime}-\lambda \rho(x) u=0, \quad \lambda>0, \quad x \in(0, l), \\
u^{\prime}(0) \cos \alpha-\left(p u^{\prime \prime}\right)(0) \sin \alpha=0, \\
u(0) \cos \beta+\left(p u^{\prime \prime}\right)^{\prime}(0) \sin \beta=0, \\
u^{\prime}(l) \cos \gamma+\left(p u^{\prime \prime}\right)(l) \sin \gamma=0, \\
u(l) \cos \delta-\left(p u^{\prime \prime}\right)^{\prime}(l) \sin \delta=0,
\end{gathered}
$$

where $p$ and $\rho$ are piecewise continuous, positive functions on $[0, l]$ and $0 \leqslant \alpha, \beta, \gamma, \delta \leqslant \pi / 2$ unless specified otherwise. We consider throughout the nonzero eigenvalues and their corresponding eigenfunctions. It is know that the

Received by the editors April 16, 1973 and, in revised form, December 17, 1973.

AMS (MOS) subject classifications (1970). Primary 34B25, 34C10; Secondary 34C20, $70 \mathrm{~J} 10$.

Key words and phrases. Eigenvalue, eigenfunctions, oscillation theory, fourth order linear differential equations, boundary value problems, vibrations, beams, Prufer transformation, comparison theorems. 
$n$th eigenvalue of (1.1), (1.2) is given by $\lambda_{n}=R\left[u_{n}\right]$ where $R$ is the Rayleigh quotient (A.1) and $u_{n}$ is the $n$th eigenfunction. It follows by inspection of the numerator of $R$ that zero is an eigenvalue only in the following cases:

(i) $\alpha=\gamma=0$ and $\beta=\delta=\pi / 2$.

(ii) Any three of $\alpha, \beta, \gamma, \delta$ equal to $\pi / 2$.

Unless stated otherwise, we exclude these cases from consideration.

In the process of our study, we also derive some oscillatory properties of solutions of the fourth order equation (1.1) with $\lambda=1$. However, we will be primarily interested in those fourth order nonsingular problems which arise from the theory of vibrating beams (see e.g. [2, Chapter 1]).

We will use a transformation of the system (1.1), (1.2) analogous to the Prüfer transformation

$$
y(x)=r(x) \sin \theta(x), \quad P(x) y^{\prime}(x)=r(x) \cos \theta(x), \quad x \in[0, \eta]
$$

used to investigate the Sturm-Liouville system

$$
\begin{array}{rlrl}
\left(P y^{\prime}\right)^{\prime}+\lambda Q y & =0, & & x \in(0, l), \\
y(0) \cos \alpha-P(0) y^{\prime}(0) \sin \alpha=0, & & \alpha \in[0, \pi / 2] \\
y(l) \cos \beta+P(l) y^{\prime}(l) \sin \beta & =0, & & \beta \in[0, \pi / 2] .
\end{array}
$$

Application of the transformation (*) to this system yields

$$
\theta^{\prime}=p^{-1} \cos ^{2} \theta+\lambda Q \sin ^{2} \theta \text { with } \theta(0)=\alpha \text { and } \theta(l)=\beta+k \pi,
$$

where $k$ is some nonnegative integer. From properties of the solution $\theta$ of this system and (*) it is possible to obtain oscillatory properties of the eigenfunctions of the Sturm-Liouville system (see e.g. [5, pp. 394-407]).

In a previous paper [1], a procedure for the computation of eigenvalues of (1.1), (1.2) was developed based on a transformation of the vector

$$
U=\left(u, u^{\prime}, p u^{\prime \prime},\left(p u^{\prime \prime}\right)^{\prime}\right)^{T}
$$

into four-dimensional spherical coordinates. Here, we use the following transformation which takes advantage of the pairings of the function $u$ and its derivatives found in the boundary conditions (1.2):

$$
\begin{aligned}
& u(x)=r(x) \sin \psi(x) \cos \theta(x), \quad p(x) u^{\prime \prime}(x)=r(x) \cos \psi(x) \cos \varphi(x), \\
& u^{\prime}(x)=r(x) \cos \psi(x) \sin \varphi(x), \quad\left(p(x) u^{\prime \prime}(x)\right)^{\prime}=r(x) \sin \psi(x) \sin \theta(x) .
\end{aligned}
$$

Assuming the validity of this transformation, we derive a set of differential equations which must be satisfied by the functions $r, \psi, \theta, \varphi$. We first write (1.1) as the equivalent first order system $U^{\prime}=M U$ where $U$ is defined by (1.3) and 


$$
M=\left[\begin{array}{cccc}
0 & 1 & 0 & 0 \\
0 & 0 & 1 / p & 0 \\
0 & 0 & 0 & 1 \\
\lambda \rho & 0 & 0 & 0
\end{array}\right] .
$$

Transforming this first order system by (1.4), and letting $w=\cot \psi$, we obtain

$$
r^{\prime}=1 / 2 r\left[\sin 2 \psi \sin (\theta+\varphi)+p^{-1} \cos ^{2} \psi \sin 2 \varphi+\lambda \rho \sin ^{2} \psi \sin 2 \theta\right]
$$

and

$$
w^{\prime}=-w^{2} \cos \theta \sin \varphi+1 / 2 p^{-1} w \sin 2 \varphi+\sin \theta \cos \varphi-1 / 2 \lambda \rho w \sin 2 \theta,
$$

$$
\theta^{\prime}=-w \sin \theta \sin \varphi+\lambda \rho \cos ^{2} \theta \text {, }
$$

$$
\varphi^{\prime}=p^{-1} \cos ^{2} \varphi-w^{-1} \sin \theta \sin \varphi \text {. }
$$

Using the transformed system, we study the properties of $\theta$ and $\varphi$ to obtain the oscillatory behavior of the eigenfunctions and their derivatives of the system (1.1), (1.2). The paper is organized as follows.

In $\S 2$, we use a result of Leighton and Nehari [9] to show that, for the solutions of (1.1) to be considered here, the Jacobian of the transformation (1.4) is not zero for $x \in(0, l)$, justifying the validity of this transformation. In $\S 3$, we give an argument which justifies the use of (1.4) at $x=0$ and $x=l$ even though the Jacobian may vanish there and determine boundary conditions for these angles at 0 and $l$.

We are not able to show the existence of eigenvalues using the differential system for the angles, as in the case for the usual Prüfer transformation, and must rely on the variational characterization of the eigenvalues. This is given in the appendix along with some consequences of this characterization which we use in the ensuing development.

In $\S 4$, we consider solutions of (1.1) which satisfy $(1.2 a, b, c)$ and prove a fundamental comparison theorem which shows the dependence of the corresponding $\theta(l)$ on the coefficients of (1.1). This in turn is used to show that $\theta(l, \lambda)$ is a strictly increasing continuous function of $\lambda$. It is also used to obtain a comparison theorem for the number of zeros of solutions of (1.1) with $\lambda=1$.

In $\S 5$, we use this comparison theorem to investigate the graph of $\theta$ for $x \in\left[0, l\right.$. From this graph, we show that the $n$th eigenfunction $u_{n}$ of (1.1), (1.2) has exactly $(n-1)$ zeros in $(0, l)$ and that $\left(p u_{n}^{\prime \prime}\right)^{\prime}$ has exactly $n$ zeros in $[0, l]$. Furthermore, the zeros of $u_{n}$ and $\left(p u_{n}^{\prime \prime}\right)^{\prime}$ interlace.

In $\S 6$, we investigate the graph of $\varphi$. From this we show that $u_{1}^{\prime}$ has at most one zero in $(0, l)$ and that $u_{n}^{\prime}$ for $n \geqslant 2$ has at least $n-2$ and at 
most $n$ zeros in $(0, l)$. Furthermore, $u_{n}^{\prime \prime}$ has at least $n-1$ and at most $n+1$ zeros in $[0, l]$. Other results concerning the interlacing of the zeros of $u_{n}$ and its derivatives are also given.

2. The transformed system. Our first consideration must be whether or not the transformation (1.4) is well defined. We note that the Jacobian of this transformation is $J=r^{3} \sin \psi \cos \psi$. This vanishes whenever $r=0$ or $\psi$ is an integer multiple of $\pi / 2$. If $r\left(x_{0}\right)=0$ for any $x_{0} \in[0, l],(1.4)$ implies that $u\left(x_{0}\right)=u^{\prime}\left(x_{0}\right)=p u^{\prime \prime}\left(x_{0}\right)=\left(p u^{\prime \prime}\right)^{\prime}\left(x_{0}\right)=0$. In this case the only solution is the trivial solution. For an arbitrary solution it may happen that $\psi\left(x_{0}\right)=$ $k \pi / 2, k=0, \pm 1, \pm 2, \cdots$, for some $x_{0} \in(0, l)$ and consequently that $J\left(x_{0}\right)=0$. However, we will show in Theorem 2.1 that, for the eigenvalue problems being considered here, this cannot occur. The following lemmas will be needed throughout our discussion.

LEMma 2.1 (LEIGHTON AND NeHARI [9]). Let $y$ be a nontrivial solution of (1.1). If $y, y^{\prime}, y^{\prime \prime},\left(p y^{\prime \prime}\right)^{\prime}$ are nonnegative (but not all zero) at $x_{0}$, then they are positive for $x>x_{0}$. If $y,-y^{\prime}, y^{\prime \prime},-\left(p y^{\prime \prime}\right)^{\prime}$ are nonnegative (but not all zero) at $x_{0}$, they are positive for $x<x_{0}$.

We will find it convenient to use the following consequence of this lemma.

LEMMA 2.2. Let $u$ be a solution of (1.1) which satisfies the boundary conditions (1.2a) and (1.2c). If $x_{0}$ is a zero of $u$ or $u^{\prime \prime}$ in $(0, l)$, then $u^{\prime}(x)\left(p u^{\prime \prime}\right)^{\prime}(x)<0$ in a neighborhood of $x_{0}$. If $x_{1}$ is a zero of $u^{\prime}$ or $\left(p u^{\prime \prime}\right)^{\prime}$ in $(0, l)$ then $u(x) u^{\prime \prime}(x)<0$ in a neighborhood of $x_{1}$.

Proof. Let $u\left(x_{0}\right)=0, x_{0} \in(0, l)$ and assume $u^{\prime}\left(x_{0}\right)\left(p u^{\prime \prime}\right)^{\prime}\left(x_{0}\right) \geqslant 0$. If $u^{\prime}\left(x_{0}\right) \geqslant 0$, then $\left(p u^{\prime \prime}\right)^{\prime}\left(x_{0}\right) \geqslant 0$. When $u^{\prime \prime}\left(x_{0}\right) \geqslant 0$, Lemma 2.1 implies that $u, u^{\prime}, p u^{\prime \prime}$, and $\left(p u^{\prime \prime}\right)^{\prime}$ are strictly positive at $l$. The boundary condition (1.2c) implies that $u^{\prime}(l)\left(p u^{\prime \prime}\right)(l) \leqslant 0$, a contradiction. When $u^{\prime \prime}\left(x_{0}\right)<0$, Lemma 2.1 yields a contradiction to the boundary condition (1.2a). A similar contradiction is reached if $u^{\prime}\left(x_{0}\right)<0$.

The same type of argument yields the remainder of the lemma.

We now consider the Jacobian of the transformation (1.4) for $x \in(0, l)$.

THEOREM 2.1. Let $u$ be a nontrivial solution of (1.1) which satisfies the boundary conditions (1.2a) and (1.2c). Then the Jacobian $J[u]=r^{3} \sin \psi \cos \psi$ of the transformation (1.4) does not vanish in $(0, l)$.

Proof. Let $u$ be a nontrivial solution of (1.1) which satisfies the boundary conditions (1.2a) and (1.2c). Assume first that the corresponding angle $\psi$ satisfies $\psi\left(x_{0}\right)=n \pi$ for some integer $n$ and for some $x_{0} \in(0, l)$. Then the transformation (1.4) implies that $u\left(x_{0}\right)=0$ and $\left(p u^{\prime \prime}\right)^{\prime}\left(x_{0}\right)=0$. However, this 
is incompatible with the conclusion of Lemma 2.2.

Now assume for the solution $u$, that the corresponding angle $\psi$ satisfies $\psi\left(x_{0}\right)=m \pi / 2$ for some odd integer $m$ and some $x_{0} \in(0, l)$. The transformation (1.4) then implies that $u^{\prime}\left(x_{0}\right)=0$ and $p u^{\prime \prime}\left(x_{0}\right)=0$. Lemma 2.2 with these conditions yields a contradiction.

COROLlary. If $u$ satisfies the hypothesis of Theorem 2.1, then the corresponding values of $w=\cot \psi$ satisfy either the condition $0<w(x)<\infty$ or $-\infty<w(x)<0$ for $x \in(0, l)$.

One consequence of this result is that there is no loss of generality in choosing $\psi(x) \in(0, \pi / 2)$ or $\psi(x) \in(\pi / 2, \pi)$ for $x \in(0, l)$.

We now consider the possibility that the Jacobian vanishes at $x=0$ or $x=l$. One such possibility occurs if $\psi=k \pi(k=0, \pm 1, \pm 2, \cdots)$ at $x=0$ or $x=l$. The following theorem shows this does not happen.

THEOREM 2.2. Let $u$ satisfy the hypothesis of Theorem 2.1. Then the corresponding value of $\psi$ at $x=0$ and $x=l$ is not an integral multiple of $\pi$.

Proof. If $\psi(0)=k \pi$, then (1.2a) implies that $u^{\prime}(0)$ and $u^{\prime \prime}(0)$ have the same sign and (1.4) implies that $u(0)=\left(p u^{\prime \prime}\right)^{\prime}(0)=0$. Then Lemma 2.1 yields a contradiction as in the proof of Lemma 2.2.

If $\psi(l)=k \pi$, then (1.2c) implies that $u^{\prime}(l)$ and $u^{\prime \prime}(l)$ have opposite sign. But (1.4) yields $u(l)=\left(p u^{\prime \prime}\right)^{\prime}(l)=0$ and Lemma 2.1 again gives a contradiction.

Finally, we must consider the possibility that $\psi$ has value $\pi / 2$ at 0 or l. At zero, $\psi(0)=\pi / 2$ gives $u^{\prime}(0)=p u^{\prime \prime}(0)=0$. It is possible to construct, using a fundamental set of solutions of (1.1), a solution of (1.1) which satisfies the boundary conditions $u^{\prime}(0)=p u^{\prime \prime}(0)=0,(1.2 \mathrm{~b})$ and $(1.2 \mathrm{c})$. Thus $\psi(0)=$ $\pi / 2$ cannot be excluded.

In the next section, where the transformation of the boundary conditions is considered, we show how this case can be handled.

3. Boundary conditions. Before we can use the differential system to analyze the behavior of $\psi, \theta, \varphi$ for a given eigenfunction, we must transform the boundary conditions (1.2). For the transformed variables $\varphi, \theta$ and $w$ at $x=0$, we obtain:

THEOREM 3.1. Let $u$ be a solution of (1.1) which satisfies the boundary conditions $(1.2 \mathrm{a}, \mathrm{b}, \mathrm{c})$. Then

$$
\theta(0)=\beta-\pi / 2
$$

and

$$
\varphi(0)=\alpha
$$


where $\alpha=0$ when $\psi(0)=\pi / 2$. Furthermore, $w(0)=\cot \psi(0)$ is determined by at least one of the following:
(a) $w(0)=\frac{u^{\prime}(0) \sin \beta}{u(0) \sin \alpha}$,
(c) $w(0)=\frac{\left(p u^{\prime \prime}\right)(0) \sin \beta}{u(0) \cos \alpha}$,
(b) $w(0)=\frac{-\left(p u^{\prime \prime}\right)(0) \cos \beta}{\left(p u^{\prime \prime}\right)^{\prime}(0) \cos \alpha^{\prime}}$,
(d) $w(0)=\frac{-u^{\prime}(0) \cos \beta}{\left(p u^{\prime \prime}\right)^{\prime}(0) \sin \alpha}$.

Proof. To find $\varphi(0)$ and $\theta(0)$, we note that the boundary conditions (1.2a) and (1.2b) under transformation (1.4) become $r_{0} \cos \psi(0) \sin (\varphi(0)-\alpha)=$ 0 and $r_{0} \sin \psi(0) \cos (\theta(0)-\beta)=0$, respectively. Since $r_{0} \neq 0$ and $\psi(0) \neq$ 0 or $\pi$, then $\theta(0)=\beta+(2 m+1) \pi / 2(m=0, \pm 1, \pm 2, \cdots)$. If $\psi(0) \neq \pi / 2$, then $\varphi(0)=\alpha+k \pi(k=0, \pm 1, \pm 2, \cdots)$. It can be seen from (1.4) that if we choose $\cos \theta(0) \geqslant 0$ and $\sin \varphi(0) \geqslant 0$, then the sign of $u(0)$ and $u^{\prime}(0)$ determines the initial value of $\psi$. Thus, we may choose $k=0$ and $m=-1$ to obtain (3.1) and (3.2).

If $\psi(0)=\pi / 2$, then $u^{\prime}(0)=u^{\prime \prime}(0)=0$. We show that in this case $\varphi(0)=$ $\alpha=0$. From (1.4),

$$
\tan \varphi(0)=\lim _{x \rightarrow 0} \frac{u^{\prime}(x)}{p u^{\prime \prime}(x)}=\lim _{x \rightarrow 0} \frac{u^{\prime \prime}(x)}{\left(p u^{\prime \prime}\right)^{\prime}(x)}=0
$$

since $\left(p u^{\prime \prime}\right)^{\prime}(0) \neq 0$ by Lemma 2.1 . Hence, we have $\alpha=0$.

To find the initial value of $w=\cot \psi$ for the given solution $u$ of (1.1), we use those pairs of equations in (1.4) whose ratio yields the $\cot \psi$ together with (3.1) and (3.2). All of these relations (3.3) are needed due to the fact that for some cases, a given ratio may be indeterminant. That at least one relation will not be indeterminant can be established from Lemma 2.1 and the boundary conditions (1.2).

For $\theta$ and $\varphi$ at $x=l$, we have:

THEOREM 3.2. Let $u$ be a solution of (1.1) which satisfies the boundary conditions (1.2a, c, d). Then

$$
\theta(l)=(2 m-1) \pi / 2-\delta \quad(m=0,1,2, \cdots),
$$

and

$$
\varphi(l)=k \pi-\gamma \quad(k=1,2, \cdots),
$$

where $\gamma=0$ when $\psi(l)=\pi / 2$.

These conclusions follow as in the proof of Theorem 3.1 for integer values of $k$ and $m$.

It is necessary to choose $k \geqslant 1$ in the expression for $\varphi(l)$ since the 
differential equation (1.5) shows that $\varphi^{\prime}>0$ whenever $\varphi=0$ for any $x \in$ $(0, l)$. By (3.2), $\varphi(0)=\alpha \geqslant 0$ so that $\varphi(l)>0$. A similar argument shows that $m \geqslant 0$.

To complete the discussion of the initial conditions which are required to determine the nature of the solutions of the angular system (1.5), we must give the case where $\psi(0)=\pi / 2$ special consideration. We have

THEOREM 3.3. Let $u$ be a solution of (1.1) which satisfies the boundary conditions $(1.2 \mathrm{a}, \mathrm{c})$ such that the corresponding function $\psi$ satisfies $\psi(0)=\pi / 2$. Then

(i) $\psi(x) \in(\pi / 2, \pi), x \in(0, l)$, and

(ii) the corresponding function $\varphi$ satisfies $\lim _{x \rightarrow 0} \varphi^{\prime}(x)=1 / 2 p(0)$.

Proof. Since $\psi(0)=\pi / 2, \alpha=0$ and from (1.5) we have $w^{\prime}(0)=$ $-\cos \beta$. But

$$
w^{\prime}(x)=\frac{d}{d x} \cot \psi(x)=-\left(\csc ^{2} \psi(x)\right) \psi^{\prime}(x)
$$

so that $\psi^{\prime}(0)>0$. Hence (i) follows from Theorem 2.1 .

For (ii), we note that when $\psi(0)=\pi / 2,1 / w(0)=\tan \psi(0)$ and hence $\varphi^{\prime}(0)$ in (1.5) is not defined. However, we do have

$$
\lim _{x \rightarrow 0} \varphi^{\prime}(x)=\frac{1}{p(0)}-\lim _{x \rightarrow 0} \frac{\sin \theta \sin \varphi}{w} .
$$

Using (1.4), and the fact that $\varphi(0)=0$ and $\left(p u^{\prime \prime}\right)^{\prime}(0) \neq 0$ (by Lemma 2.1), we find that

$$
\begin{aligned}
\lim _{x \rightarrow 0} \frac{\sin \theta \sin \varphi}{w} & =\lim _{x \rightarrow 0}\left[\frac{\left(p u^{\prime \prime}\right)^{\prime}}{p u^{\prime \prime}}\right]^{2} \frac{u^{\prime}}{\left(p u^{\prime \prime}\right)^{\prime}} \cos ^{2} \varphi \\
& =\left(p u^{\prime \prime}\right)^{\prime}(0) \lim _{x \rightarrow 0} \frac{u^{\prime}}{\left(p u^{\prime \prime}\right)^{2}}=\frac{1}{2 p(0)} .
\end{aligned}
$$

The conclusion (ii) follows.

REMARK. Since a similar argument holds when $\psi(l)=\pi / 2$, it follows that there is a uniquely determined direction field for the system (1.5) in all cases.

4. Comparison theorems. We are now able to prove the following comparison theorem.

THEOREM 4.1. Let $y_{0}$ and $y_{1}$ be solutions of

$$
\left(p_{0} y_{0}^{\prime \prime}\right)^{\prime \prime}-\rho_{0} y_{0}=0
$$

and 


$$
\left(p_{1} y_{1}^{\prime \prime}\right)^{\prime \prime}-\rho_{1} y_{1}=0
$$

respectively for $x \in(0, l)$, both of which satisfy the boundary conditions (1.2a, $b, c)$. Let $0<\rho_{0}(x) \leqslant \rho_{1}(x)$ and $p_{0}(x) \geqslant p_{1}(x)>0$ for $x \in[0, l]$. Furthermore, let $\theta_{0}$ and $\theta_{1}$ be the $\theta$-angles corresponding to $y_{0}$ and $y_{1}$ respective$l y$ as determined by the transformation (1.4). Then $\theta_{0}(l) \leqslant \theta_{1}(l)$ with equality if and only if $\rho_{0} \equiv \rho_{1}$ and $p_{0} \equiv p_{1}$ on $[0, l]$.

Proof. Let $\rho_{\mu}=\mu \rho_{1}+(1-\mu) \rho_{0}$ and $p_{\mu}=\mu p_{1}+(1-\mu) p_{0}$, where $0 \leqslant \mu \leqslant 1$, so that $\rho_{\mu}$ is nondecreasing and $p_{\mu}$ is nonincreasing as $\mu$ increases. We consider a solution $y_{u}$ of the system determined by the equation

$$
\left(p_{\mu} y^{\prime \prime}\right)^{\prime \prime}-\rho_{\mu} y=0
$$

and the three boundary conditions $(1.2 \mathrm{a}, \mathrm{b}, \mathrm{c})$. Let $\theta_{\mu}$ and $\varphi_{\mu}$ be the corresponding angles determined by the transformation (1.4). By (3.4), the angle $\theta_{\mu}(x)$ at $x=l$ gives

$$
\delta_{\mu}=(2 m-1) \pi / 2-\theta_{\mu}(l)
$$

where $m$ is such that $0 \leqslant \delta_{\mu}<\pi$. We assume first that $\delta_{\mu} \neq 0$. That the solutions of the system (1.5) with initial conditions (3.1), (3.2), (3.3) depend continuously on the parameter $\mu$ follows from the theory of ordinary differential equations. We show that $\theta_{\mu}(l)$ is a strictly increasing function of $\mu$, so that $\theta_{1}(l) \geqslant \theta_{0}(l)$ with equality if and only if $\rho_{0}=\rho_{1}$ and $p_{0}=p_{1}$. To establish that $\theta_{\mu}(l)$ is increasing we use (4.4) and prove that $\delta_{\mu} \geqslant \delta_{\nu}$ where $0 \leqslant \mu<$ $\nu \leqslant 1$.

Now consider the eigenvalue problem consisting of

$$
\left(p_{\mu} u^{\prime \prime}\right)^{\prime \prime}-\lambda \rho_{\mu} u=0
$$

and the boundary conditions $(1.2)$ with $\delta \in(0, \pi)$. The existence and variational characterization of the eigenvalues of this system are discussed in the appendix.

Denote the $n$th eigenvalue of this system by $\lambda_{n}[\mu, \delta]$. In particular, the solution $y_{\mu}$ of (4.3) must be an eigenfunction of (4.5) corresponding to $\lambda_{N}\left[\mu, \delta_{\mu}\right]=1$ for some integer $N$.

We next consider a solution $y_{\nu}$ of (4.3), (1.2a, b, c) where $\mu$ has been replaced by $\nu$. The corresponding angular system (1.5) with the initial conditions (3.1), (3.2), (3.3) with $\mu$ replaced by $\nu,(\nu>\mu)$ yields the angle $\theta_{\nu}(l)$. By continuity, we may take $\nu-\mu$ so small that

$$
\delta_{\nu}=(2 m-1) \pi / 2-\theta_{\nu}(l)
$$

where $m$ is the integer determined by $(4.4)$ and $\delta_{\nu} \in(0, \pi)$. In particular, the solution $y_{\nu}$ must be an eigenfunction of (4.5) with corresponding eigenvalue $\lambda_{M}\left[\nu, \delta_{\nu}\right]=1$ for some integer $M$. But the continuity of eigenvalues as a 
function of the parameter $\mu$ implies that $\lambda_{N}\left[\mu, \delta_{\mu}\right]$ and $\lambda_{M}\left[\nu, \delta_{\nu}\right]$ are both the $n$th eigenvalue of the system (4.5) for $|\nu-\mu|$ sufficiently small.

Now consider the $n$th eigenvalue $\lambda_{n}\left[\nu, \delta_{\mu}\right]$ of (4.5), (1.2) where we have taken $\delta=\delta_{\mu}$ in (1.2) and replaced $\mu$ by $\nu$ in (4.5). By the comparison theorem for eigenvalues (see the appendix, properties 2 and 3)

$$
\lambda_{n}\left[\nu, \delta_{\mu}\right] \leqslant \lambda_{n}\left[\mu, \delta_{\mu}\right]=1
$$

with equality if and only if $\rho_{0} \equiv \rho_{1}$ and $p_{0} \equiv p_{1}$. From the max-min characterization of eigenvalues (see appendix), $\lambda_{n}[\nu, \delta]$ is a strictly decreasing continuous function of $\delta$. Thus the inequality $\delta_{\nu}>\delta_{\mu}$ implies that $\lambda_{n}\left[\nu, \delta_{\nu}\right]<$ $\lambda_{n}\left[\nu, \delta_{\mu}\right]$, and (4.7) yields $\lambda_{n}\left[\nu, \delta_{\nu}\right]<1$, a contradiction. Hence $\delta_{\nu} \leqslant \delta_{\mu}$ and, by (4.4) and (4.6), $\theta_{\nu}(l) \geqslant \theta_{\mu}(l)$ with equality if and only if $\rho_{0} \equiv \rho_{1}$ and $p_{0} \equiv p_{1}$.

Finally, we consider those values $\left\{\mu_{0}\right\}$ for which $\theta_{\mu_{0}}(l)=(2 m-1) \pi / 2$, corresponding to $\delta_{\mu_{0}}=0$. By the continuity of $\theta_{\mu}(l)$ with respect to $\mu$ and the established monotonicity of $\theta_{\mu}(l)$ in a deleted neighborhood of $\mu_{0}, \theta_{\mu}(l)$ is monotone for $0 \leqslant \mu \leqslant 1$. Hence $\theta_{0}(l) \leqslant \theta_{1}(l)$.

From this theorem, we get immediately

THEOREM 4.2. Let $u$ be a solution of equation (1.1) which satisfies the boundary conditions $(1.2 \mathrm{a}, \mathrm{b}, \mathrm{c})$ and let $\theta(x, \lambda), x \in[0, l]$ be the corresponding $\theta$-angle determined by the transformation (1.4). Then $\theta(l, \lambda)$ is a strictly increasing continuous function of $\lambda$.

Theorem 4.1 can also be used to obtain comparison theorems of the following type.

THEOREM 4.3. Let $u$ be a solution of (4.1) such that $u(0)=u^{\prime}(0)=$ $u(l)=u^{\prime}(l)=0$ and let $v$ be a solution of (4.2) such that $v(0)=v^{\prime}(0)=v(l)=$ $v^{\prime}(l)=0$ where the coefficients in (4.1) and (4.2) are related as stated in Theorem 4.1. Then the number of zeros of $v$ in $(0, l)$ is greater than the number of zeros of $u$ unless $\rho_{0} \equiv \rho_{1}$ and $p_{0} \equiv p_{1}$.

5. Properties of $\theta$ and consequences. In this section, we use the comparison theorem on $\theta(l, \lambda)$ to show that the $n$th eigenfunction $u_{n}$ of the system (1.1), (1.2) has exactly $n-1$ zeros in $(0, l)$ and that $\left(p u_{n}^{\prime \prime}\right)^{\prime}$ has exactly $n$ zeros in $[0, l]$. Our procedure for obtaining these results will be to establish properties of the graph of $\theta$ corresponding to the $n$th eigenfunction $u_{n}$.

Our first two theorems are devoted to showing that the graph of $\theta$ intersects the lines $\theta=k \pi$ and $\theta=(k+1 / 2) \pi, k$ an integer, with positive slope.

THEOREM 5.1. Let $u$ be a nontrivial solution of (1.1) which satisfies (1.2a) 
and (1.2c). Then the graph of the corresponding function $\theta$ for $x \in(0, l)$ intersects the lines $\theta=m \pi(m=0,1,2, \cdots)$ with positive slope.

Proof. From (1.5), $\theta^{\prime}=\lambda \rho>0$ when $\theta=m \pi$ so that the first conclusion holds.

THEOREM 5.2. Let $u$ be a solution of (1.1) which satisfies the boundary conditions (1.2a) and (1.2c). Then the corresponding graph of $\theta$ on $(0, l)$ can only intersect the lines $\theta=(2 m-1) \pi / 2$ with positive slope, $m=0,1, \cdots$.

Proof. Let $x_{1}$ be a point in $(0, l)$ such that $\theta\left(x_{1}\right)=(2 k+1) \pi / 2$ for $k=0,1,2, \cdots$ and assume that $\theta^{\prime}\left(x_{1}\right) \leqslant 0$. From (1.4) we know that

$$
u^{\prime}\left(x_{1}\right) /\left(p u^{\prime \prime}\right)^{\prime}\left(x_{1}\right)=w(x) \sin \varphi\left(x_{1}\right) / \sin \theta\left(x_{1}\right) .
$$

From (1.5),

$$
\theta^{\prime}\left(x_{1}\right)=-w\left(x_{1}\right) \sin \theta\left(x_{1}\right) \sin \varphi\left(x_{1}\right)
$$

Consequently,

$$
u^{\prime}\left(x_{1}\right) /\left(p u^{\prime \prime}\right)^{\prime}\left(x_{1}\right)=-\theta^{\prime}\left(x_{1}\right) / \sin ^{2} \theta\left(x_{1}\right)=-\theta^{\prime}\left(x_{1}\right) .
$$

Since $\theta^{\prime}\left(x_{1}\right) \leqslant 0, u^{\prime}\left(x_{1}\right)$ and $\left(p u^{\prime \prime}\right)^{\prime}\left(x_{1}\right)$ have the same sign at $x_{1}$. But $u\left(x_{1}\right)=$ 0 , contradicting Lemma 2.2 .

THEOREM 5.3. Let $u$ be a solution of (1.1) which satisfies the boundary conditions (1.2a) and (1.2c). Then $\theta(x)>-\pi / 2$ for $x \in(0, l)$.

Proof. Suppose that $u(x)>0$ in a deleted neighborhood $N$ of $x=0$. Then (1.4) and $r \sin \psi>0$ implies that $\cos \theta>0$. Hence, $\theta(x) \in(-\pi / 2, \pi / 2)$ in $N$. Let $x_{1}$ be the first point (if there is one) in $(0, l)$ such that $\theta\left(x_{1}\right)=$ $-\pi / 2$. Since $\theta(x)>-\pi / 2$ for $x<x_{1}$ and $\theta^{\prime}\left(x_{1}\right) \neq 0$ by Theorem 5.1, $0>\theta^{\prime}\left(x_{1}\right)$, contradicting Theorem 5.2.

If $u(0)>0$, then $u(x)>0$ in $N$. If $u(0)=0$ and $u^{\prime}(0) \neq 0$, then the homogeneity of (1.1) permits us to assume that $u^{\prime}(0)>0$ and hence that $u(x)>0$ in $N$. Finally, if $u(0)=u^{\prime}(0)=0$, we may take $u^{\prime \prime}(0)>0$. Integrating twice, we find that $u(x)>0$ in $N$. Since this exhausts all possible cases we have $\theta(x)>-\pi / 2$ in $(0, l)$.

Note that this shows that $m=0$ is not an admissible choice of $m$ in (3.4). The following theorem will lead to the major results of this section.

THEOREM 5.4. Let $0<\lambda_{1}<\lambda_{2}<\cdots<\lambda_{n}<\cdots$ denote the ordered eigenvalues of the system (1.1), (1.2). Let $\theta(l, \lambda), \lambda>0$, denote the value of the $\theta$-angle at $x=l$ determined by (1.4) and corresponding to the solution $u(x, \lambda)$ of (1.1) which satisfies the boundary conditions (1.2a, b, c). Then 


$$
\theta\left(l, \lambda_{n}\right)=(2 n-1) \pi / 2-\delta, \quad n=1,2, \cdots .
$$

Proof. We first consider the special case $\delta=\pi / 2$ so that (1.2d) becomes $\left(p u^{\prime \prime}\right)^{\prime}(l)=0$ and assume that $\lambda_{1} \neq 0$ in this case. We show that $\theta\left(l, \lambda_{1}\right)=0$. By (3.1), $-\pi / 2 \leqslant \theta(0) \leqslant 0$. Furthermore, the condition $\left(p u^{\prime \prime}\right)^{\prime}(l)=0$ with (1.4) implies $\sin \theta\left(l, \lambda_{1}\right)=0$. But from the differential equation (1.5), for all values of $x$ for which $\sin \theta=0$, we have $\theta^{\prime}(x)>0$. This with (3.4) implies $\theta\left(l, \lambda_{1}\right) \geqslant 0$.

We show the reverse inequality holds by use of our comparison theorem.

Let $p_{0}$ and $\rho_{0}$ be positive constants such that $p_{0} \leqslant p(x)$ and $\rho_{0} \geqslant \rho(x)$ for $x \in[0, l]$ and consider the system

$$
p_{0} y^{(\mathrm{iv})}-\mu \rho_{0} y=0, \quad(1.2 \mathrm{a}, \mathrm{b}, \mathrm{c}) \text { and } y^{\prime \prime \prime}(\mathrm{l})=0 .
$$

We first assume that zero is not an eigenvalue of (5.2) so that the Green's function of $L y=d^{4} y / d x^{4}$ with the stated boundary conditions exists. In [8], Kreith shows that the Green's function is positive when the boundary conditions are given by (1.2) with $\alpha, \beta, \gamma, \delta$ in $(0, \pi / 2)$. This result can be extended to apply to the system (5.2) by continuity considerations. It now follows from Jentzsch's theorem [7] that the first eigenfunction $y_{1}$ of $(5.2)$ does not vanish in $(0, l)$.

Let $\theta^{*}$ be the $\theta$-angle corresponding to this eigenfunction. It follows that the value of $\theta^{*}$ at $x=l$ is zero. For otherwise $\theta^{*}(l) \geqslant \pi$ and at some interior point $\theta^{*}$ would have the value $\pi / 2$. This would imply that $u$ is zero at an interior point, a contradiction.

By our comparison theorem (Theorem 4.1), the angle $\theta\left(l, \lambda_{1}\right)$ corresponding to the system (1.1), (1.2a, b, c) and the angle $\theta^{*}(l)$ corresponding to the system (5.2) satisfy the inequality $\theta\left(l, \lambda_{1}\right) \leqslant \theta^{*}(l)=0$. Hence $\theta\left(l, \lambda_{1}\right)=0$ when $\delta=\pi / 2$ and $\lambda_{1} \neq 0$.

If the assumption made at the beginning of the proof is not valid, i.e., that the system consisting of $(1.1),(1.2 \mathrm{a}, \mathrm{b}, \mathrm{c})$ and $\left(p u^{\prime \prime}\right)^{\prime}(l)=0$ has a first eigenvalue $\lambda_{1}=0$, then we note that $u_{1}(x)$ is a linear function. It follows from (1.4) that $\sin \theta \equiv 0$. Hence $\theta=k \pi$ for some integer and $\theta(0) \in[-\pi / 2,0]$ then yields $\theta(x) \equiv 0$. Thus $\theta\left(l, \lambda_{1}\right)=0$ also in this special case.

Now consider any $\delta \in[0, \pi / 2)$. Then by property 1 of the appendix, the first eigenvalue $\lambda_{1}$ of $(1.1),(1.2)$ with $\delta \in[0, \pi / 2]$ is greater the the lowest eigenvalue of the same system with $\delta=\pi / 2$. We have just shown that the $\theta$ angle determined by the eigenfunction corresponding to the lowest eigenvalue with $\delta=\pi / 2$ is zero at $x=l$. Since by Theorem $4.2, \theta(l, \lambda)$ is a strictly increasing continuous function of $\lambda$, it follows that there is a number $\lambda_{1}$ such that $\theta\left(l, \lambda_{1}\right)=\pi / 2-\delta$. As noted in the appendix (property 4 ), $\lambda_{1}$ must be a simple zero of $\theta(l, \lambda)=\pi / 2-\delta$. 
For the second eigenvalue $\lambda_{2}$ of (1.1), (1.2), we note that (3.4) is satisfied. Theorem 4.2 thus implies that $\theta\left(l, \lambda_{2}\right)=3 \pi / 2-\delta$. Finally, (5.1) follows by induction on $n$, using the fact that the eigenvalues are simple.

We note that this result also applies to the eigenvalues $\lambda_{n}$ for $n \geqslant 2$ in those exceptional cases where $\lambda_{1}=0$ except where $\alpha=\beta=\gamma=\delta=\pi / 2$ for which $\lambda_{1}=\lambda_{2}=0$.

As a direct consequence of Theorem 5.4, we have:

THEOREM 5.5. Under the hypothesis of Theorem 5.4, the nth eigenfunction $u_{n}$ of (1.1), (1.2) has at least $n-1$ zeros in $(0, l)$ and $\left(p u_{n}^{\prime \prime}\right)^{\prime}$ has exactly $n$ zeros in $[0, l]$.

We now proceed to show that the $n$th eigenfunction $u_{n}$ of (1.1), (1.2) has exactly $n-1$ zeros in $(0, l)$. This will be a consequence of the following theorem which summarizes the results of Theorems 5.1, 5.2, 5.3 and 5.4.

THEOREM 5.6. Let $u_{n}$ be the nth eigenfunction of the system (1.1), (1.2) and let $\theta$ be the corresponding angular function determined by (1.4). Let $\xi_{k}$ denote the values $x$ where $\theta\left(\xi_{k}\right)=k \pi(k=0,1,2, \cdots, n-1)$ and let $\eta_{k}$ denote those values where $\theta\left(\eta_{k}\right)=(2 k+1) \pi / 2(k=0,1,2, \cdots, n-2)$. Then

$$
0 \leqslant \xi_{0}<\eta_{0}<\xi_{1}<\eta_{1}<\cdots<\eta_{n-2}<\xi_{n-1} \leqslant l .
$$

In terms of the $n$th eigenfunction, we have that $u_{n}\left(\eta_{k}\right)=0$ and $\left(p u_{n}^{\prime \prime}\right)^{\prime}\left(\xi_{k}\right)=0$. Thus, Theorem 5.6 yields:

THEOREM 5.7. Let $u_{n}$ be the nth eigenfunction of the system (1.1), (1.2). Then $u_{n}$ has exactly $n-1$ zeros $\eta_{k}$ in $(0, l)$ and $\left(p u_{n}^{\prime \prime}\right)^{\prime}$ has exactly $n$ zeros $\xi_{k}$ in $[0, l]$. These zeros are interlaced as indicated by (5.3).

6. Properties of $\varphi$ and consequences. Results for $u_{n}^{\prime}$ and $u_{n}^{\prime \prime}$ and the corresponding graph of $\varphi$ analogous to the results of $\S 5$ will be given in this section. Before discussing the zeros of $u_{n}^{\prime}$ and $u_{n}^{\prime \prime}$, we give results concerning the graph of $\varphi$. Corresponding to Theorem 5.1, we have:

THEOREM 6.1. Let $u$ be a solution of (1.1) which satisfies (1.2a) and (1.2c). Then the graph of corresponding function $\varphi$ on $(0, l)$ intersects the lines $\varphi=m \pi(m=0,1,2, \cdots)$ with positive slope.

The proof parallels that of Theorem 5.1 and will not be given.

THEOREM 6.2. Let $u$ be a solution of (1.1) which satisfies the boundary conditions (1.2a) and (1.2c). Then the corresponding graph of $\varphi$ on $(0, l)$ can only intersect the lines $\varphi=(2 k+1) \pi / 2$ with positive slope for $k=0,1,2, \cdots$. 
Proof. Let $x_{1}$ be a point in $(0, l)$ such that $\varphi\left(x_{1}\right)=(2 k+1) \pi / 2$ for some $k=0,1,2, \cdots$ and assume that $\varphi^{\prime}\left(x_{1}\right) \leqslant 0$. As in the proof of Theorem 5.3, we use (1.4) and (1.5) to get

$$
u^{\prime}\left(x_{1}\right) /\left(p u^{\prime \prime}\right)^{\prime}\left(x_{1}\right)=-\left(w\left(x_{1}\right) / \sin \theta\left(x_{1}\right)\right)^{2} \varphi^{\prime}\left(x_{1}\right) \geqslant 0 .
$$

Since $u^{\prime}\left(x_{1}\right)$ and $\left(p u^{\prime \prime}\right)^{\prime}\left(x_{1}\right)$ then have the same sign, we conclude, as in the proof of Theorem 5.3, that $u$ cannot satisfy the conditions (1.2a) and (1.2c).

As a corollary, we have:

COROLLARY. Let $u$ be a solution of (1.1) which satisfies the boundary conditions (1.2a) and (1.2c). Then there is exactly one simple zero of $u^{\prime \prime}$ between consecutive zeros of $u^{\prime}$.

We now prove analogs of Theorem 5.4. These results are stated in Theorems 6.4 and 6.5. We first establish an upper bound for $\varphi\left(l, \lambda_{n}\right)$.

LEMMA 6.1. Let $\lambda_{n}$ be the nth eigenvalue of the system (1.1), (1.2) and let $\varphi\left(l, \lambda_{n}\right)$ denote the value of the corresponding angle $\varphi$ at $x=l$ determined by (1.4). Then

$$
\varphi\left(l, \lambda_{n}\right) \leqslant(n+1) \pi-\gamma .
$$

Proof. By (2.7), we have $\varphi\left(l, \lambda_{n}\right)=k \pi-\gamma$ where $k$ is some positive integer. Suppose $\varphi\left(l, \lambda_{n}\right) \geqslant(n+2) \pi-\gamma$. Then $p u^{\prime \prime}$ has at least $n+2$ zeros which correspond to the points where the graph of $\varphi\left(x, \lambda_{n}\right)$ crosses the lines $\varphi=(2 m+1) \pi / 2, m=0,1, \cdots, n+1$. Thus $\left(p u_{n}^{\prime \prime}\right)^{\prime}$ has at least $n+1$ zeros in $(0, l)$ which contradicts Theorem 5.5.

The inequality (6.1) with $n=1$ and Theorem 6.1 show that there may be two possibilities, i.e., $\varphi\left(l, \lambda_{1}\right)=\pi-\gamma$ or $\varphi\left(l, \lambda_{1}\right)=2 \pi-\gamma$. We note that both cases are necessary since the boundary conditions for the clamped-free beam lead to the first case with $\gamma=\pi / 2$ and the boundary conditions for the clampedclamped beam lead to the second case with $\gamma=0$. Thus, there is no simple result analogous to Theorem 5.4 which gives the value of $\varphi\left(l, \lambda_{n}\right)$.

We next show the interrelationship between the function $\theta$ and $\varphi$ corresponding to a solution $u_{n}$ of the differential equation (1.1) which satisfies the boundary conditions (1.2). This relationship will depend on the algebraic sign of $w(0)=\cot \psi(0)$, that is, whether $\psi(0) \in(0, \pi / 2)$ or $\psi(0) \in[\pi / 2, \pi)$. We show that the initial conditions on $u_{n}$ determine the appropriate range of $\psi$.

THEOREM 6.3. If (i) $u_{n}(0) u_{n}^{\prime}(0)>0$, (ii) $u_{n}(0)=0$, or (iii) $u_{n}^{\prime}(0)=0$ and $u_{n}(0) u_{n}^{\prime \prime}(0)>0$, then

$$
\psi(x) \in(0, \pi / 2) \text { for } x \in(0, l) .
$$




$$
\begin{aligned}
& \text { If (iv) } u_{n}(0) u_{n}^{\prime}(0)<0 \text {, (v) } u_{n}^{\prime}(0)=0 \text { and } u_{n}(0) u_{n}^{\prime \prime}(0)<0 \text {, or (vi) } \\
& u_{n}^{\prime}(0)=u_{n}^{\prime \prime}(0)=0, \text { then }
\end{aligned}
$$

$$
\psi(x) \in(\pi / 2, \pi) \text { for } x \in(0, l) .
$$

Proof. Cases (i) and (iv) follow directly from (3.3a) and Theorem 2.1. Cases (iii) and (v) follow from (3.3c) and Theorem 2.1. From (1.4), case (vi) corresponds to $\psi(0)=\pi / 2$. It was shown in $\S 2$ that this implies that $\psi(x) \in$ $(\pi / 2, \pi)$ for $x \in(0, l)$. For case (ii), assume $\psi(0) \in[\pi / 2, \pi)$ and note that (1.4) implies that $\theta(0)=-\pi / 2$. Consequently, (1.4) yields $u_{n}^{\prime}(0) \leqslant 0, u_{n}^{\prime \prime}(0) \leqslant$ 0 and $\left(p u_{n}^{\prime \prime}\right)^{\prime}(0)<0$. Lemma 2.1 then leads to the conclusion that $u^{\prime}(l) u^{\prime \prime}(l)>$ 0 , which is incompatible with the boundary condition (1.2c).

To show the realtionship between the functions $\theta$ and $\varphi$ corresponding to an eigenfunction $u_{n}$ of (1.1), (1.2) we must consider each of the two cases $\psi(x) \in(0, \pi / 2)$ and $\psi(x) \in(\pi / 2, \pi), x \in(0, l)$ and establish bounds on the values of $\varphi$ at points in $(0, l)$ where $\theta(x)=m \pi / 2(m=0,1, \cdots, 2 n-2)$ for each case. Lemma 6.2 gives lower bounds for these values. It will be necessary to derive the consequences of these bounds before upper bounds can be determined.

LEMMA 6.2. Let $\theta, \varphi$ and $\psi$ be functions corresponding by (1.4) to the nth eigenfunction $u_{n}$ of the system (1.1), (1.2). Let $\xi_{k}$ and $\eta_{k}$ be such that $\theta\left(\xi_{k}\right)=k \pi$ and $\theta\left(\eta_{k}\right)=(k+1 / 2) \pi$ where $\xi_{k}$ and $\eta_{k}$ are related as in (5.4). If $\psi(x) \in(0, \pi / 2), x \in(0, l)$ then $\varphi\left(\xi_{k}\right)>(2 k+1) \pi / 2 \quad(k=0,1,2, \cdots$, $n-1)$ and $\varphi\left(\eta_{k}\right)>(k+1) \pi(k=0,1,2, \cdots, n-2)$. If $\psi(x) \in(\pi / 2, \pi)$, $x \in(0, l)$, then $\varphi\left(\xi_{0}\right) \geqslant 0$ and $\varphi\left(\xi_{k}\right)>(2 k-1) \pi / 2(k=1,2, \cdots, n-1)$ while $\varphi\left(\eta_{k}\right)>k \pi(k=0,1,2, \cdots, n-2)$.

Proof. We first show that if $\psi(0) \in(0, \pi / 2)$ then $\xi_{0}>0$. For if $\xi_{0}=$ 0 , then $\theta(0)=0$. Since $\varphi(0)=\alpha$, the use of (1.4) and Lemma 2.1 leads to a contradiction of the boundary condition (1.2c).

Now consider the case where $\psi \in(0, \pi / 2)$ and let $\xi_{0} \in(0, l)$ be such that $\theta\left(\xi_{0}, \lambda_{n}\right)=0$ so that $\left(p u_{n}^{\prime \prime}\right)^{\prime}\left(\xi_{0}\right)=0$. From $(1.4), u_{n}\left(\xi_{0}\right)=r\left(\xi_{0}\right) \sin \psi\left(\xi_{0}\right)>0$. Consequently Lemma 2.2 implies that $\left(p u_{n}^{\prime \prime}\right)\left(\xi_{0}\right)=r\left(\xi_{0}\right) \cos \psi\left(\xi_{0}\right) \cos \varphi\left(\xi_{0}\right)<0$. Thus, $\cos \varphi\left(\xi_{0}\right)<0$. Since $\cos \varphi(0, \lambda)=\cos \alpha>0$, Theorem 6.1 implies that $\varphi\left(\xi_{0}\right)>\pi / 2$.

Now let $\eta_{0}$ be such that $\theta\left(\eta_{0}\right)=\pi / 2$. Then from (1.4), $u_{n}\left(\eta_{0}\right)=0$ so that by Lemma 2.2, $u_{n}^{\prime}\left(\eta_{0}\right)\left(p u_{n}^{\prime \prime}\right)^{\prime}\left(\eta_{0}\right)<0$. However, by (1.4), $\left(p u_{n}^{\prime \prime}\right)^{\prime}\left(\eta_{0}\right)>0$ so that $u_{n}^{\prime}\left(\eta_{0}\right)<0$ and consequently $\sin \varphi\left(\eta_{0}\right)<0$. Since $\eta_{0}>\xi_{0}$ and $\varphi\left(\eta_{0}\right)>\pi / 2$, Theorem 6.2 implies that $\varphi\left(\eta_{0}\right)>\pi$.

Now assume that $\eta_{k-1}$ is such that $\theta\left(\eta_{k-1}\right)=(k-1 / 2) \pi$ and $\varphi\left(\eta_{k-1}\right)$ $\geqslant k \pi$ for some $k \geqslant 1$. Let $\xi_{k}$ be such that $\theta\left(\xi_{k}\right)=k \pi$. Then from (1.4), 
$\left(p u_{n}^{\prime \prime}\right)^{\prime}\left(\xi_{k}\right)=0$ so Lemma 2.2 implies that $u_{n}\left(\xi_{k}\right)\left(p u_{n}^{\prime \prime}\right)\left(\xi_{k}\right)<0$. But the sign of $u_{n}\left(\xi_{k}\right)$ is the same as the sign of $\cos k \pi=(-1)^{k}$. If $k$ is odd, then $p u_{n}^{\prime \prime}\left(\xi_{k}\right)$ $>0$ and (1.4) with $\psi \in(0, \pi / 2)$ implies that $\cos \varphi\left(\xi_{k}\right)>0$. Hence, $\xi_{k}>\eta_{k-1}$ and $\varphi\left(\eta_{k-1}\right) \geqslant k \pi$ yield $\varphi\left(\xi_{k}\right)>(k+1 / 2) \pi$ (k odd). A similar argument yields this result for $k$ even.

Now let $\eta_{k}$ be such that $\theta\left(\eta_{k}\right)=(k+1 / 2) \pi$ where $\eta_{k}>\xi_{k}$ and $\varphi\left(\xi_{k}\right)$ $>(k+1 / 2) \pi$. From (1.4), $u_{n}\left(\eta_{k}\right)=0$ so that Lemma 2.2 implies that $u_{n}^{\prime}\left(\eta_{k}\right)\left(p u_{n}^{\prime \prime}\left(\eta_{k}\right)\right)^{\prime}<0$. But (1.4) shows that the sign of $\left(p u_{n}^{\prime \prime}\left(\eta_{k}\right)\right)^{\prime}$ is the same as $\sin (k+1 / 2) \pi=(-1)^{k}$. If $k$ is odd, then $\left(p u_{n}^{\prime \prime}\right)^{\prime}\left(\eta_{k}\right)<0$ and $u_{n}^{\prime}\left(\eta_{k}\right)=$ $r \cos \psi\left(\eta_{k}\right) \sin \varphi\left(\eta_{k}\right)>0$. But $\psi \in(0, \pi / 2)$ implies $\sin \varphi\left(\eta_{k}\right)>0$. From this, along with $\eta_{k}>\xi_{k}, \varphi\left(\xi_{k}\right)>(k+1 / 2) \pi$ and Theorem 6.2, we conclude that $\varphi\left(\eta_{k}\right)>(k+1) \pi$. A similar argument gives the same result when $k$ is even.

The above argument holds for $k=1,2, \cdots, n-2$. To complete the proof for the case $\psi(x) \in(0, \pi / 2)$, we repeat for $k=n-1$ the argument which shows that $\varphi\left(\xi_{k}\right)>(2 k+1) \pi / 2$.

For the case $\psi(x) \in[\pi / 2, \pi)$, we note that $\varphi\left(\xi_{0}\right) \geqslant 0$ from Theorem 6.1 and the fact that $\varphi(0)=\alpha$. We apply the argument used in the case $\psi(x) \in$ $(0, \pi / 2)$. For $\xi_{0}$ (with special consideration in case $\xi_{0}=0$ ), we are only able to conclude that $\varphi\left(\xi_{0}\right) \geqslant 0$ again. For $\eta_{0}$, we are only able to conclude that $\varphi\left(\eta_{0}\right)>0$. Finally, for $\eta_{k}(k=1,2, \cdots, n-2)$ and $\xi_{k}(k=1,2, \cdots$, $n-1)$, we find, using the arguments of the previous case, that $\varphi\left(\eta_{k}\right)>k \pi$ and $\varphi\left(\xi_{k}\right)>(2 k-1) \pi / 2$.

We use this lemma to prove the following:

THEOREM 6.4. Let $u_{n}$ be the $n$th eigenfunction of the eigenvalue problem (1.1), (1.2) and let $\theta, \varphi$, and $\psi$ be the corresponding angular functions determined by (1.4). If $\psi(0) \in(0, \pi / 2)$, then either $\varphi\left(l, \lambda_{n}\right)=n \pi-\gamma$ or $\varphi\left(l, \lambda_{n}\right)=$ $(n+1) \pi-\gamma$.

Proof. We have that $\varphi\left(\xi_{n-1}\right) \geqslant(2 n-1) \pi / 2, \xi_{n-1} \leqslant l$, from Lemma 6.2. From Lemma 6.1 , we have that $\varphi(l) \leqslant(n+1) \pi-\gamma$. But (3.5) implies that $\varphi(l)=m \pi-\gamma$ for some integer $m$. Consequently, $m=n$ or $n+1$.

From this we immediately have the following:

COROLlaRY. Under the hypothesis of Theorem 6.4, either $u_{n}^{\prime}$ has $n-1$ zeros in $(0, l)$ interlaced with $n$ zeros of $u_{n}^{\prime \prime}$ in $[0, l]$ or $u_{n}^{\prime}$ has $n$ zeros in $(0, l)$ interlaced with $(n+1)$ zeros of $u_{n}^{\prime \prime}$ in $[0, l]$.

Both cases are possible as the following two examples show.

Consider the equation

$$
u^{(\mathrm{iv})}-\lambda u=0, \quad x \in(0, l) .
$$


For solutions of (6.3) which satisfy the boundary conditions

$$
u(0)=u^{\prime}(0)=0,
$$

we find that the corresponding value of $\psi(0)$ lies in $(0, \pi / 2)$. We first consider the $n$th eigenfunction $u_{n}$ determined by the system (6.3), (6.4) and $u(l)=$ $u^{\prime}(l)=0$. Theorem 5.7 then shows that $u_{n}$ has $n-1$ zeros in $(0, l)$. Thus $u_{n}^{\prime}$ has at least $n$ zeros in $(0, l)$. By the above corollary, it has exactly $n$ zeros in $(0, l)$ and hence $\varphi\left(l, \lambda_{n}\right)=(n+1) \pi$.

Next, consider the $n$th eigenfunction $u_{n}$ of the system (6.3), (6.4) and $u^{\prime \prime}(l)=u^{\prime \prime \prime}(l)=0$. By the same argument used above, $u_{n}^{\prime}$ has at least $n-1$ zeros in $(0, l)$. That it has no more than $n-1$ zeros is a consequence of Lemma 2.2. For $u_{n}^{\prime}\left(x_{m}\right)=0$ implies that $u_{n}\left(x_{m}\right) u_{n}^{\prime \prime}\left(x_{m}\right)<0$ so that all such points $x_{m}$ are relative extrema and there is only one such point between zeros of $u_{n}$. Thus $\varphi\left(l, \lambda_{n}\right)=n \pi$ in this case.

When $\psi(0) \in[\pi / 2, \pi)$, we prove

THEOREM 6.5. Let $u_{n}$ be the eigenfunction given in the hypothesis of Theorem 6.4. If $\psi(0) \in[\pi / 2, \pi)$, then $\varphi\left(l, \lambda_{1}\right)=\pi-\gamma$ and for $n \geqslant 2$, either $\varphi\left(l, \lambda_{n}\right)=n \pi-\gamma$ or $\varphi\left(l, \lambda_{n}\right)=(n-1) \pi-\gamma$.

Proof. By (3.5), $\varphi\left(l, \lambda_{1}\right) \geqslant \pi-\gamma$. Hence, by Lemma 6.1, $\varphi\left(l, \lambda_{1}\right)$ is either $2 \pi-\gamma$ or $\pi-\gamma$. For $n \geqslant 2$, Lemma 6.2 and (3.5) yield $\varphi\left(l, \lambda_{n}\right) \geqslant$ $(n-1) \pi-\gamma$. This and Lemma 6.1 then imply that $\varphi\left(l, \lambda_{n}\right)$ has one of the values $(n+1) \pi-\gamma, n \pi-\gamma$, or $(n-1) \pi-\gamma$ for $n \geqslant 2$. We show that $\psi(0) \in[\pi / 2, \pi)$ precludes the possibility that $\varphi\left(l, \lambda_{n}\right)=(n+1) \pi-\gamma, n=$ $1,2, \cdots$.

Assume that $\varphi\left(l, \lambda_{n}\right)=(n+1) \pi-\gamma$. Then $\varphi\left(0, \lambda_{n}\right)=\alpha \in[0, \pi / 2]$ implies the existence of an increasing sequence of numbers $\left\{x_{m}\right\}, m=1, \cdots$, $n$ in $(0, l)$ such that $\varphi\left(x_{m}, \lambda_{n}\right)=m \pi$. By $(1.4), u_{n}^{\prime}\left(x_{m}\right)=0$. As in the previous theorem, Lemma 2.2 shows that in each of the intervals $\left(x_{m}, x_{m+1}\right)$, $m=1,2, \cdots, n-1$, there is one zero of $u_{n}$. Furthermore, since $\psi(0) \in$ $[\pi / 2, \pi)$, Theorem 6.3 implies that $u_{n}(0)>0$.

But at $x_{1},(1.4)$ implies that $u_{n}^{\prime \prime}\left(x_{1}\right)>0$ and Lemma 2.2 implies that $u_{n}\left(x_{1}\right)<0$. Hence, $u_{n}$ has a zero in $\left(0, x_{1}\right)$ and at least $n$ zeros in $(0, l)$ contradicting Theorem 5.7.

From Theorem 6.5 , we have the following:

COROLlary. Under the hypothesis of Theorem 6.5, the first eigenfunction $u_{1}$ has the property that $u_{1}^{\prime}$ has no zeros in $(0, l)$ and $u_{1}^{\prime \prime}$ has one zero in $[0, l]$. If $n \geqslant 2$, either $u_{n}^{\prime}$ has $n-2$ zeros in $(0, l)$ interlaced with $n-1$ zeros of $u_{n}^{\prime \prime}$ in $[0, l]$ or $u_{n}^{\prime}$ has $n-1$ zeros in $(0, l)$ interlaced with $n$ zeros of $u_{n}^{\prime \prime}$ in $[0, l]$. 
That Theorem 6.5 has the strongest possible conclusion is easily seen by considering the $n$th eigenvalues of the systems consisting of (6.3) with boundary condition $u^{\prime}(0)=u^{\prime \prime \prime}(0)=u^{\prime}(l)=u^{\prime \prime \prime}(l)=0$ and (6.3) with boundary condition $u^{\prime}(0)=u^{\prime \prime \prime}(0)=u(l)=u^{\prime \prime}(l)=0$. The first system has the solutions

$$
u_{n}(x)=\cos \frac{(n-1) \pi x}{l}
$$

and consequently $\varphi\left(l, \lambda_{n}\right)=(n-1) \pi$ for $n \geqslant 2$. The second system has solution

$$
u_{n}(x)=\cos \frac{(2 n-1) \pi x}{2 l}
$$

and $\varphi\left(l, \lambda_{n}\right)=(2 n-1) \pi / 2=n \pi-\pi / 2, n=1,2, \cdots$.

The results of Theorems 6.4 and 6.5 together with the method of proof of Lemma 6.2 can be used to establish the upper bounds for $\varphi\left(\xi_{k}\right)$ and $\varphi\left(\eta_{k}\right)$ previously mentioned.

LEMMA 6.3. Let $\theta, \varphi$, and $\psi$ correspond by (1.4) to the nth eigenfunction of (1.1), (1.2). Let $\theta\left(\xi_{k}\right)=k \pi$ and $\theta\left(\eta_{k}\right)=(2 k+1) \pi / 2$ where $\xi_{k}$ and $\eta_{k}$ are related as in (5.3).

If $\psi(x) \in(0, \pi / 2)$, then $\varphi\left(\eta_{k}\right)<(k+2) \pi(k=0,1, \cdots, n-2)$. If, in addition,

(i) $\varphi(l)=n \pi-\gamma$, then $\varphi\left(\xi_{k}\right)<(2 k+3) \pi / 2 \quad(k=0,1, \cdots, n-2)$ with $\varphi\left(\xi_{n-1}\right) \leqslant n \pi$;

while $n-1)$.

(ii) $\varphi(l)=(n+1) \pi-\gamma$ implies $\varphi\left(\xi_{k}\right)<(2 k+3) \pi / 2 \quad(k=0,1, \cdots$,

If $\psi(x) \in[\pi / 2, \pi)$ and $\varphi(l)=n \pi-\gamma$, then

(iii) $\varphi\left(\xi_{k}\right) \leqslant(2 k+1) \pi / 2 \quad(k=0,1, \cdots, n-1)$ with equality possible only when $k=0$. Also $\varphi\left(\eta_{k}\right)<(k+1) \pi \quad(k=0,1, \cdots, n-2)$.

If $\psi(x) \in[\pi / 2, \pi)$ and $\varphi(l)=(n-1) \pi-\gamma, n \geqslant 2$, then

(iv) $\varphi\left(\eta_{k}\right)<(k+2) \pi$ and $\varphi\left(\xi_{k}\right)<(2 k+1) \pi / 2(k=0,1, \cdots, n-2)$ with $\varphi\left(\xi_{n-1}\right)<(n-1) \pi$.

Since the proof of this lemma is essentially the same as the proof of Lemma 6.2 , we indicate how the proof of case (iii) starts and leave the remainder of the proof to the reader.

Partial proof. We first note that $\cos \theta(l)=(-1)^{n} \sin \delta$ and $\sin \theta(l)=$ $(-1)^{n} \cos \delta$ by (5.1). For case (iii), $\varphi(l)=n \pi-\gamma$ so that $\cos \theta(l)=$ $(-1)^{n+1} \sin \gamma$ and $\cos \varphi(l)=(-1)^{n} \cos \gamma$. At $\xi_{n-1}$, we have $\theta\left(\xi_{n-1}\right)=$ $(n-1) \pi$ so that $\cos \theta\left(\xi_{n-1}\right)=(-1)^{n+1}$ and by $(1.4),\left(p u_{n}^{\prime \prime}\right)^{\prime}\left(\xi_{n-1}\right)=0$. By the usual argument, these conditions imply by Lemma 2.1 that $\xi_{n-1}<l$. 
By Lemma 2.2 we have $u_{n}\left(\xi_{n-1}\right) u_{n}^{\prime \prime}\left(\xi_{n-1}\right)<0$ so that the value of $\sin \psi \cos \psi \cos \theta \cos \varphi$ is negative at $x=\xi_{n-1}$. Since $\psi\left(\xi_{n-1}\right) \in(\pi / 2, \pi)$, we have $\cos \theta\left(\xi_{n-1}\right) \cos \varphi\left(\xi_{n-1}\right)>0$. But $\cos \theta\left(\xi_{n-1}\right)=(-1)^{n+1}$ yields $\cos \varphi\left(\xi_{n-1}\right)=(-1)^{n+1} A$ for some positive number $A$. From $\cos \varphi(l)=$ $(-1)^{n} \cos \gamma$, it follows that $\varphi\left(\xi_{n-1}\right)<(2 n-1) \pi / 2$. The proof is completed by applying this type of argument successively at the points $\eta_{n-2}>\xi_{n-2}>\cdots$ $>\eta_{0}>\xi_{0} \geqslant 0$.

From Lemmas 6.2 and 6.3, we obtain the following theorems which give some of the oscillatory properties of $u_{n}$ and its derivatives. When $\psi(x) \in$ $(0, \pi / 2)$, we have

THEOREM 6.6. Let $u_{n}$ be the nth eigenfunction of the system (1.1), (1.2) such that $\psi(x) \in(0, \pi / 2)$ and denote the consecutive zeros of $u_{n}$ by $\eta_{0}, \eta_{1}$, $\cdots, \eta_{n-2}$ and the consecutive zeros of $\left(p u_{n}^{\prime \prime}\right)^{\prime}$ by $\xi_{0}, \xi_{1}, \cdots, \xi_{n-1}$. Then

(i) In $\left(\eta_{k}, \eta_{k+\nu}\right), u_{n}^{\prime}$ has exactly $\nu$ zeros, $k \geqslant 0, k+\nu \leqslant n-2$.

(ii) In $\left(\xi_{k}, \xi_{k+\nu}\right), u_{n}^{\prime \prime}$ has exactly $\nu$ zeros, $k \geqslant 0, k+\nu \leqslant n-1$.

(iii) In $\left(\eta_{k}, \eta_{k+\nu}\right), u_{n}^{\prime \prime}$ has at least $\nu-1$ and at most $\nu+1$ zeros with $k \geqslant 0, k+\nu \leqslant n-2$.

(iv) In $\left(\xi_{k}, \xi_{k+\nu}\right), u_{n}^{\prime}$ has at least $\nu-1$ and at most $\nu+1$ zeros, $k \geqslant 0, k+\nu \leqslant n-1$.

(v) In $\left[0, \xi_{0}\right), u_{n}^{\prime \prime}$ has exactly one zero. In $\left(\xi_{n-1}, l\right], u_{n}^{\prime \prime}$ has no zeros if $\varphi(l)=n \pi-\gamma$, and exactly one zero if $\varphi(l)=(n+1) \pi-\gamma$.

For $\psi(x) \in[\pi / 2, \pi)$, we have the corresponding:

THEOREM 6.7. Let $u_{n}$ be the nth eigenfunction of the system (1.1), (1.2) such that $\psi(x) \in[\pi / 2, \pi)$ and denote the zeros of $u_{n}$ and $\left(p u_{n}^{\prime \prime}\right)^{\prime}$ as above. Then for $n \geqslant 2$, statements (i), (ii) and (iii) of Theorem 6.6 hold. Statement (iv) holds for $k \geqslant 1$ but in $\left(\xi_{0}, \xi_{1}\right), u_{n}^{\prime}$ has one zero or none. Statement (v) must be replaced by:

(v') In $\left(0, \xi_{0}\right), u_{n}^{\prime \prime}$ has no zeros. If $\varphi(l)=(n-1) \pi-\gamma, u_{n}^{\prime \prime}$ has no zeros in $\left(\xi_{n-1}, l\right]$, but if $\varphi(l)=n \pi-\gamma, u_{n}^{\prime \prime}$ has one zero in $\left(\xi_{n-1}, l\right]$.

\section{APPENDIX. SOME PROPERTIES OF EIGENVALUES}

In this section, we give certain properties of the eigenvalues of the system (1.1), (1.2) which were used to investigate the behavior of the functions $\theta$ and $\varphi$ obtained from the transformation (1.4).

When $\alpha, \beta, \gamma$, and $\delta$ of (1.2) lie in the interval $[0, \pi / 2]$ it is well known that the eigenvalues of (1.1), (1.2) are determined from the Rayleigh quotient

$$
R[y]=\left(\int_{0}^{l} p y^{\prime \prime 2} d x+N[y]\right) / \int_{0}^{l} \rho y^{2} d x
$$


with

(A.2) $N[y]=\left[y^{\prime}(0)\right]^{2} \cot \alpha+[y(0)]^{2} \cot \beta+\left[y^{\prime}(l)\right]^{2} \cot \gamma+[y(l)]^{2} \cot \delta$

by the max-min principle [3, p. 405]. We use the convention that if any of the parameters $\alpha, \beta, \gamma$, or $\delta$ are zero, then the boundary value of $y$ or $y^{\prime}$ at 0 or $l$ is taken to be zero and the corresponding term in $N[y]$ does not appear.

We will need this max-min characterization of the eigenvalues for some cases where $\delta$ is in the interval $[0, \pi)$. The validity of the max-min principle for this more general situation can be established using known arguments once it is shown that the Rayleigh quotient is bounded below [10, p. 247]. But it can be shown using the theory of differential operators in Hilbert space, that this is the case (see e.g. [4, p. 1454]).

With this max-min characterization, the following properties of the eigenvalues can be deduced (see [3, p. 418]).

PROPERTY 1. The eigenvalues of (1.1), (1.2) are continuous, strictly decreasing functions of $\alpha, \beta, \gamma$, and $\delta$ for $\alpha, \beta, \gamma, \delta \in(0, \pi)$.

PROPERTY 2. The eigenvalues of (1.1), (1.2) are continuous, strictly decreasing functions of the density $\rho$, i.e., if $\rho_{1} \leqslant \rho_{2}$ are two density functions with $\rho_{1}$ and $\rho_{2}$ not identical on a set of positive measure then $\lambda_{n}\left[\rho_{1}\right]>$ $\lambda_{n}\left[\rho_{2}\right]$.

Property 3. The eigenvalues of (1.1), (1.2) are continuous, strictly increasing functions of the stiffness $p$.

We will also need the following result.

PROPERTY 4. The eigenvalues of (1.1), (1.2) are simple.

For a proof, see [6].

\section{REFERENCES}

1. D. Banks and G. Kurowski, Computation of eigenvalues for vibrating beams, SIAM J. Numer. Anal. (to appear).

2. L. Collatz, Eigenwerteprobleme und ihrer numerische Behandlung, Chelsea, New York, 1948. MR 8, 514.

3. R. Courant and D. Hilbert, Methoden der mathematischen Physik. Vol. 1, Springer, Berlin, 1931; English transl., Interscience, New York, 1953. MR 16, 426.

4. N. Dunford and J. T. Schwartz, Linear operators. II: Spectral theory. Selfadjoint operators in Hilbert space, Interscience, New York, 1963. MR 32 \#6181.

5. E. Hille, Lectures on ordinary differential equations, Addison-Wesley, Reading, Mass., 1969. MR 40 \#2939.

6. S. Janczewsky, Oscillation theorems for the differential boundary value problems of the fourth order, Ann. of Math. (2) 29 (1928), 521-542.

7. R. Jentzsch, Über Integralgleichungen mit positiven kern, J. Reine Angew. Math. 141 (1912), 235-244.

8. K. Kreith, Comparison theorems for constrained rods, SIAM Rev. 6 (1964), 3136. MR $28 \# 4172$. 
9. W. Leighton and Z. Nehari, On the oscillation of solutions of self-adjoint linear differential equations of the fourth order, Trans. Amer. Math. Soc. 89 (1958), 325-377. MR 21 \#1429.

10. F. Riesz and B. Sz.-Nagy, Leçons d'analyse fonctionnelle, Akad. Kiadó, Budapest, 1953; English transl., Ungar, New York, 1955. MR 15, 132; 17, 175.

DEPARTMENT OF MATHEMATICS, UNIVERSITY OF CALIFORNIA, DAVIS, CALIFORNIA 95616 